\title{
Cifoescoliosis severa secundaria a neurofibromatosis. Presentación de un caso
}

\author{
Luis E. Nuñez Alvarado," Edgar Morales Vásquez," Raúl Macchiavello Falcon" \\ "Servicio de Ortopedia y Traumatología, Instituto Nacional de Salud del Niño - San Borja, Lima, Perú \\ ** Cirugía Ortopédica y Traumatología, Hospital Nacional Guillermo Almenara, Lima, Perú \\ ${ }^{*}$ Cirugía Ortopédica y Traumatología, Hospital Nacional María Auxiliadora, Lima, Perú
}

\begin{abstract}
RESUMEN
La escoliosis distrófica de la neurofibromatosis se caracteriza por ser una cifoescoliosis de ángulo agudo que compromete un segmento corto de la columna vertebral y genera una gran deformidad que, sumada a los cambios distróficos de la columna, convierte a los gestos quirúrgicos para su corrección en verdaderos retos. Se presenta a un varón de 15 años con cifoescoliosis toracolumbar distrófica severa con ápice en T9, ángulo de Cobb $107^{\circ}$ de escoliosis y $110,7^{\circ}$ de cifosis segmentaria. Se realiza una cirugía por vía posterior en tres tiempos, mediante una resección de la columna vertebral y reemplazo con malla de titanio; se logra una corrección de la cifosis y la escoliosis del $56 \%$ y 59,8\%, respectivamente. El paciente no tuvo complicaciones mayores, ni secuelas, y la evolución fue favorable. La resección de la columna vertebral es una técnica quirúrgica potente y desafiante para el manejo de la deformidad cifoescoliótica compleja necesaria para lograr el equilibrio espinal, aunque no está exenta de complicaciones, sobre todo neurológicas y pulmonares, a veces, inevitables. La calidad de vida de nuestro paciente tuvo una mejoría importante.
\end{abstract}

Palabras clave: Neurofibromatosis; escoliosis; resección; columna vertebral.

Nivel de Evidencia: IV

\section{Severe kyphoscoliosis secondary to neurofibromatosis. Case presentation}

\begin{abstract}
Dystrophic scoliosis in neurofibromatosis is identifiable by being an acute-angle kyphoscoliosis involving a short segment of the spine and producing severe deformity that when associated with the dystrophic changes of the spine result in real surgical challenges. We report the clinical case of a 15-year male with severe dystrophic kyphoscoliosis at the thoracolumbar area, with apex at T9, scoliosis with a Cobb angle of $107^{\circ}$, and segmental kyphosis of $110.7^{\circ}$. The patient underwent a three-stage surgery, performed through a posterior approach, involving a vertebral column resection (VCR) and titanium mesh replacement, and achieving a kyphosis correction of $56 \%$ and a scoliosis correction of $59.8 \%$. The patient experienced no major complications nor sequelae and had a favorable course. The VCR is a powerful and demanding surgical technique that allows for the management of the complex kyphoscoliosis deformity to achieve spinal balance; however, it is not without complications, especially neurological and pulmonary complications, which may be unavoidable. Our patient's quality of life has improved significantly.
\end{abstract}

Key words: Neurofibromatosis; scoliosis; resection; spine.

Level of Evidence: IV

\section{INTRODUCCIÓN}

La neurofibromatosis es una facomatosis de causa multifactorial con una base genética autosómica dominante, aunque el $50 \%$ de los casos se produce por mutaciones de novo. Se trata de una proliferación de células de la cresta neural, puede ocurrir en el sistema nervioso tanto periférico como central. ${ }^{1}$ Se expresa en cuatro formas clínicas, la más común es la neurofibromatosis tipo 1 o enfermedad de von Recklinghausen que se caracteriza por la presencia de neurofibromas diseminados en el organismo y "manchas café con leche" en la piel y otros tejidos ectodérmicos. ${ }^{2}$ La incidencia es de un caso cada 3000 nacidos vivos. ${ }^{3}$

Recibido el 19-9-2019. Aceptado luego de la evaluación el 4-12-2019 • Dr. LUIS E. NÚÑEZ ALVARADO • luisenriquenunez@ hotmail.com Cómo citar este artículo: Nuñez Alvarado LE, Morales Vásquez E, Macchiavello Falcon R. Cifoescoliosis severa secundaria a neurofibromatosis. Presentación de un caso. Rev Asoc Argent Ortop Traumatol 2020;85(4):393-402. https://doi.org/10.15417/issn. 1852-7434.2020.85.4.1015 
Las manifestaciones musculoesqueléticas incluyen: osteoporosis, osteomalacia, osteopenia, baja talla y macrocefalia; las manifestaciones ortopédicas comunes son escoliosis y seudoartrosis congénita (más común de la tibia) (Tabla). ${ }^{4}$

Tabla. Criterios diagnósticos de la neurofibromatosis tipo 1

Se necesitan dos o más de los siguientes criterios:

1. Seis o más manchas café con leche: de, al menos, $0,5 \mathrm{~cm}$ de longitud en prepúberes de, al menos, $1,5 \mathrm{~cm}$ de longitud en pospúberes

2. Dos o más neurofibromas de cualquier tipo, o uno o más neurofibromas plexiformes

3. Pecas en las axilas o ingles

4. Glioma de vías ópticas

5. Dos o más nódulos de Lisch

6. Una lesión ósea distintiva: Displasia del esfenoides

Displasia o adelgazamiento cortical del hueso largo

7. Un familiar de primer grado con neurofibromatosis tipo 1

Tomada de la cita bibliográfica 3.

En estos pacientes, la escoliosis se clasifica en distrófica y no distrófica. La escoliosis no distrófica presenta un comportamiento parecido al de la escoliosis idiopática del adolescente, pero comienza a una edad temprana (antes de los 7 años), y es probable que progrese a escoliosis distrófica. ${ }^{5}$ La escoliosis distrófica se caracteriza por el abombamiento de los cuerpos vertebrales con bordes muy afilados, la rotación vertebral muy pronunciada, el ensanchamiento del canal medular y de los forámenes vertebrales, así como el adelgazamiento costal y la presencia de masas paravertebrales. ${ }^{3}$ Predomina la cifosis sobre la escoliosis, con deformidad en ángulo agudo que incluye menos de seis segmentos vertebrales, asociados a acuñamiento vertebral. ${ }^{6}$

El manejo de las curvas distróficas debe ser agresivo; se propone la observación seriada cada seis meses solo si las curvas tienen $<20^{\circ}$ y la artrodesis vertebral posterior en curvas, $>20-40^{\circ} .{ }^{6}$ En curvas severas, se debe sopesar la colocación de un soporte anterior, y debe ser la regla en curvas cifóticas agudas, añadiendo autoinjerto y aloinjerto para evitar la seudoartrosis.

Presentamos este caso, porque no se han publicado reportes sobre el manejo de la cifoescoliosis severa en Perú, y realizamos una breve revisión de la bibliografía sobre este cuadro.

\section{CASO CLÍNICO}

Varón de 15 años, de origen étnico mestizo, estudiante, sin antecedentes familiares destacables. Acude con diagnóstico de neurofibromatosis tipo 1 . Tiene una deformidad toracolumbar, dolor dorsal que limita sus actividades diarias e incapacidad para realizar ejercicio físico a causa de la dificultad respiratoria (informa que le "falta el aire").

En el examen físico, se observa una giba toracolumbar izquierda grande y múltiples manchas café con leche en la piel (Figura 1). Los signos del talle y de Adams son positivos. Mide $1,43 \mathrm{~m}$ y pesa $39 \mathrm{~kg}$; la fuerza muscular es de 5/5. La sensibilidad bilateral está conservada y tiene reflejos osteotendinosos de miembros inferiores conservados simétricos, reflejo de Babinski bilateral indiferente, clonus negativo, reflejo abdominocutáneo simétrico y una marcha punta-talón conservada.

El puntaje total en el cuestionario de la Scoliosis Research Society-22 (SRS 22) 7 antes de la cirugía era de 2,8/5 (función 3,2/5; dolor 4,2/5; autoimagen 2,2/5; salud mental 1,8/5; satisfacción/insatisfacción con el manejo 2/5).

En la evaluación radiográfica, se observó, en el plano coronal, una escoliosis doble curva torácica mayor, con características distróficas, de ángulo agudo; un ángulo de Cobb de $107^{\circ}$ de la curva torácica principal y de $52,3^{\circ}$ de la curva torácica proximal que, a las radiografías de lateralización extrema (bendings), reducen a $92,4^{\circ}$ y $38,2^{\circ}$, respectivamente; y un balance coronal $-34,7 \mathrm{~mm}$, hombros en balance. Los hallazgos en el plano sagital fueron gran cifosis segmentaria de $110,7^{\circ}$, cifosis T4-T12 de $130,3^{\circ}$, balance sagital $+71,8 \mathrm{~mm}$, lordosis lumbar $-80,4^{\circ}$, incidencia pelviana $44,7^{\circ}$, inclinación pelviana $10,5^{\circ}$ y pendiente sacra $34,2^{\circ}$ (Figura 2 ). 


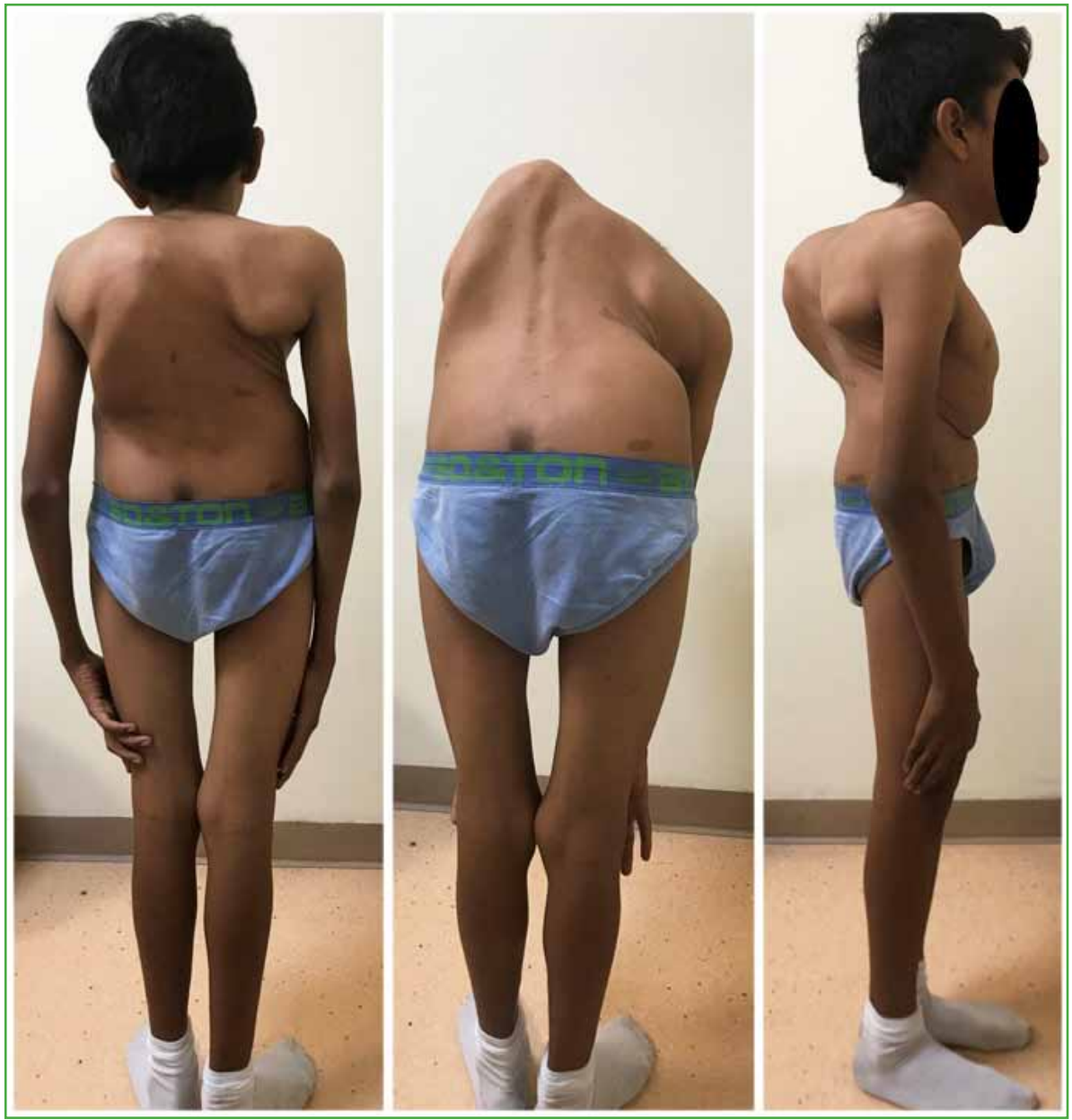

Figura 1. Imágenes clínicas del paciente, de frente, test de Adams y de perfil.
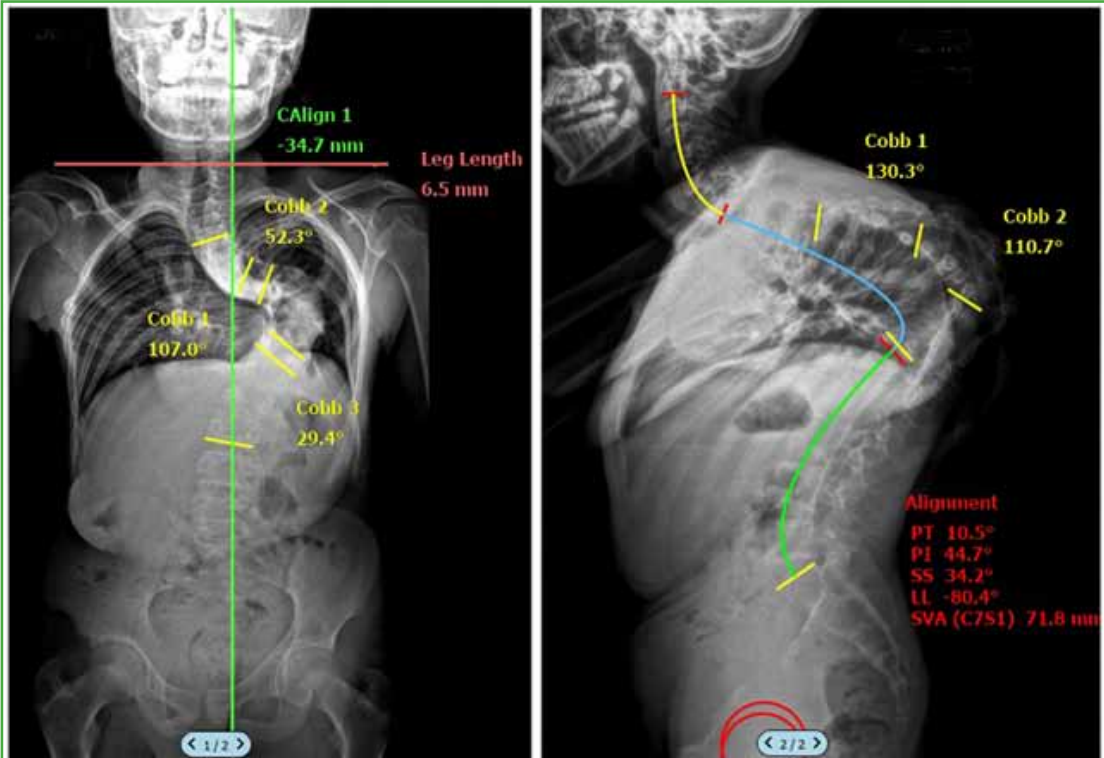

Figura 2. Espinograma prequirúrgico anteroposterior y lateral: ángulo de Cobb $107^{\circ}$, balance coronal $-34,7 \mathrm{~mm}$, cifosis segmentaria $110,7^{\circ}$, cifosis global $130,3^{\circ}$, balance sagital $71,8 \mathrm{~mm}$. 
Las imágenes por tomografía computarizada revelaron cambios distróficos característicos de la neurofibromatosis, como ensanchamiento del canal medular y de los forámenes vertebrales, adelgazamiento costal, displasia pedicular y acuñamiento vertebral. La resonancia magnética mostró la ectasia dural característica, no se observaron neurofibromas. A nivel del ápice, se observó la médula desviada hacia la concavidad (Figura 3).

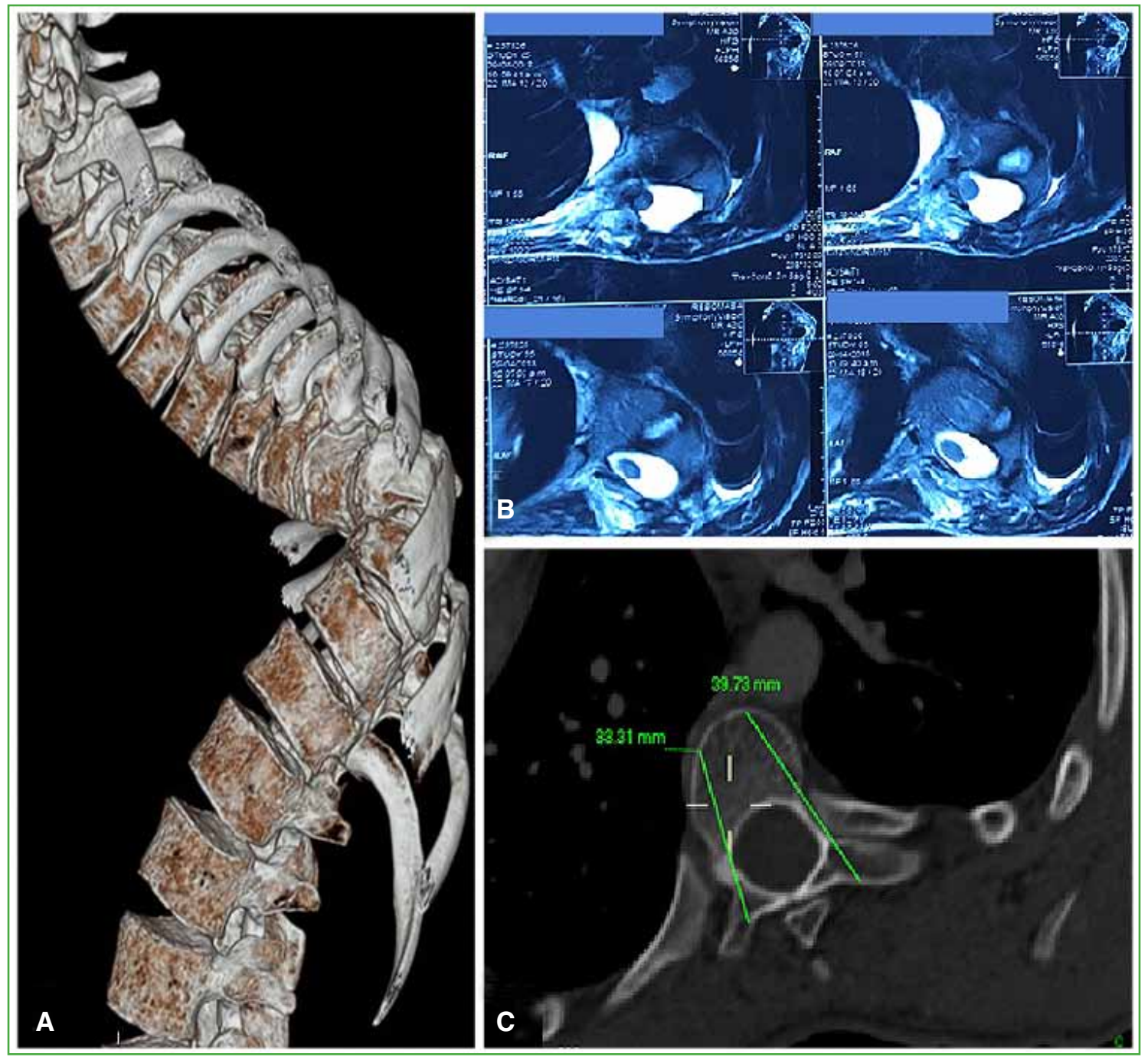

Figura 3. A y C. Tomografía espiral multicorte. Se observan el festoneado de los cuerpos vertebrales, displasia de pedículos, vértebra angulada y afinamiento de las costillas. B. Resonancia magnética. Se visualiza la ectasia dural.

Por la rigidez de la cifosis y la escoliosis, se propuso una corpectomía vertebral posterior en dos tiempos; de esta manera, se realizarían dos cirugías de menor tiempo quirúrgico y con menos sangrado por acto operatorio. El puntaje $\mathrm{FOCOS}^{8}$ de riesgo quirúrgico fue de 32, es decir, un nivel 5, lo que se traduce como un riesgo hasta del 80\% de sufrir alguna complicación mayor durante el procedimiento. La evaluación por neumología halló una incapacidad ventilatoria restrictiva grave (secundaria a la escoliosis), los datos de la espirometría fueron: volumen espiratorio forzado en 1 segundo 40\%, capacidad vital forzada 36\%. La evaluación prequirúrgica detectó un riesgo cardiológico II, un puntaje ASA II y un riesgo neumológico moderado. 
En consecuencia, se procedió al manejo quirúrgico de la enfermedad. En el primer tiempo, se realizó la instrumentación con tornillos pediculares desde T4 hasta L3 con la técnica a mano alzada, dejando libre el segmento T9-T10; se efectuaron también osteotomías de Ponte en T7-T8 y T11-T12. El primer tiempo quirúrgico duró $4 \mathrm{~h}$ y el sangrado total fue de $400 \mathrm{~cm}^{3}$. En el segundo tiempo, se inició la liberación vertebral posterior del segmento T9-T10 y la corpectomía por vía posterior de la vértebra T9, pero tuvo que suspenderse por una baja súbita de los potenciales neurofisiológicos motores, que se diagnosticó como shock medular. De inmediato, se procedió al manejo, según el protocolo NASCIS III, ${ }^{9}$ con metilprednisolona $30 \mathrm{mg} / \mathrm{kg}$ en bolo y $5,4 \mathrm{mg} / \mathrm{kg} / \mathrm{h}$ por $23 \mathrm{~h}$. Al despertar, el paciente no tenía secuelas y el puntaje ASIA era E. La segunda intervención se prolongó 7 h y 40 min, y el sangrado total fue de $1000 \mathrm{~cm}^{3}$. Durante la tercera cirugía, se terminó la corpectomía de la vértebra T9 (Figura 4) y la disectomía de T8 y T10 con reemplazo utilizando una malla de titanio rellena de autoinjerto, se colocaron autoinjerto y aloinjerto posteriores y, luego, barras. El tercer tiempo quirúrgico duró 6 h y $30 \mathrm{~min}$, con un sangrado total de $1500 \mathrm{~cm}^{3}$.

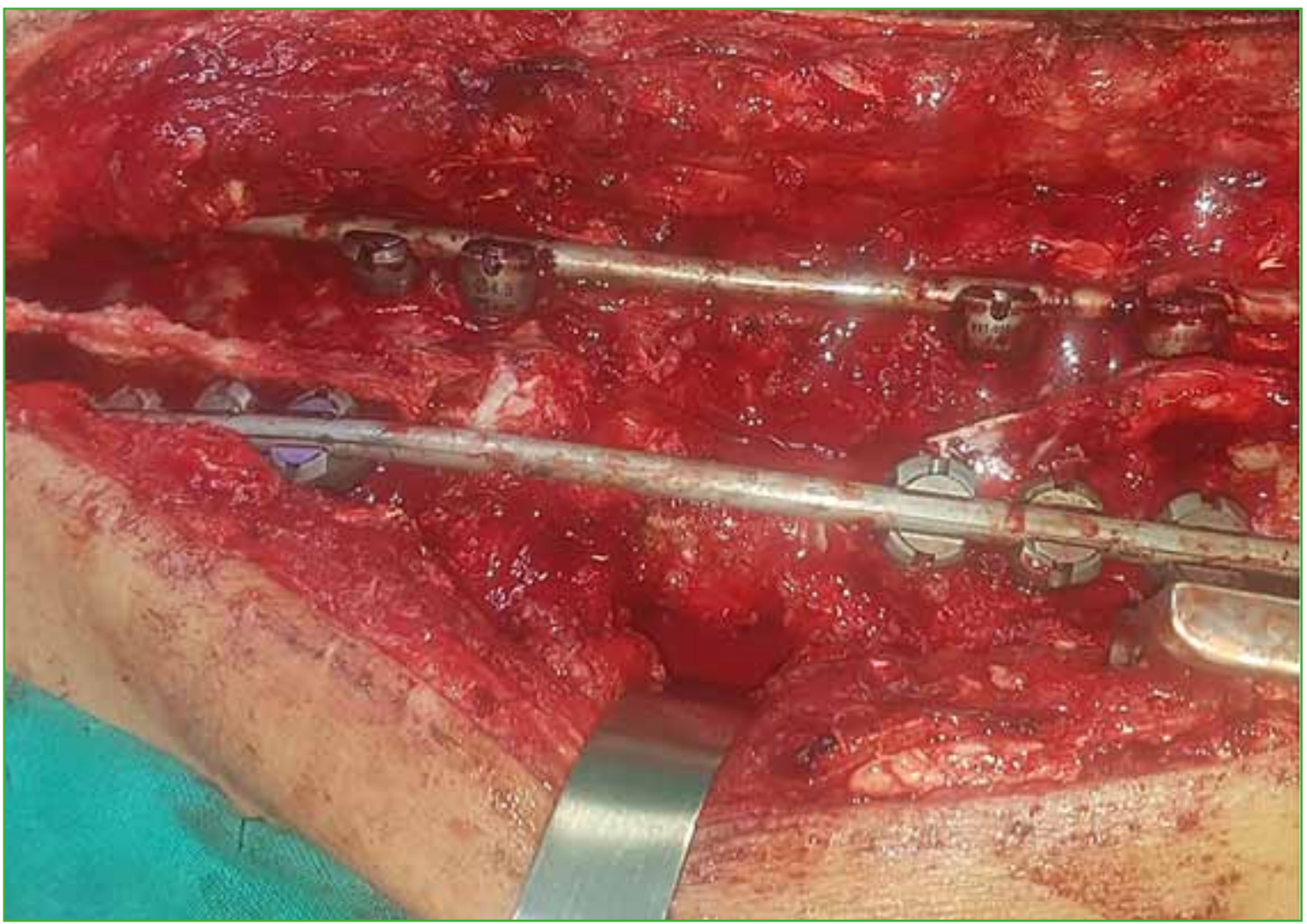

Figura 4. Defecto de continuidad en la columna vertebral luego de la resección de la columna vertebral y la corrección de la cifoescoliosis.

El tiempo total de las tres cirugías fue de $18 \mathrm{~h}$ y $10 \mathrm{~min}$, con una pérdida sanguínea total de $2900 \mathrm{~cm}^{3}$. El paciente estuvo hospitalizado 27 días, permaneció tres días en la unidad de cuidados intensivos. Usó un corsé de yeso durante seis meses después de la cirugía. A los seis meses, la tomografía mostró la integración del injerto, por lo que se decidió retirar el corsé de yeso. Al año de seguimiento, el paciente medía $159,5 \mathrm{~cm}$ y pesaba 47,8 $\mathrm{kg}$, con un índice de masa corporal de 18,7. Las medidas radiográficas posquirúrgicas fueron: ángulo de Cobb $43^{\circ}$ de la curva torácica principal y $7^{\circ}$ de la curva torácica proximal; se observó una corrección del 59,8\% y 86,6\%, respectivamente; con un balance coronal $-12,9 \mathrm{~mm}$ y hombros en balance. En el plano sagital, los hallazgos fueron: mejoría de la cifosis segmentaria a $48,6^{\circ}$, cifosis T4-T12 de $59,8^{\circ}$, balance sagital $-0,1 \mathrm{~mm}$, lordosis lumbar $69^{\circ}$, incidencia pelviana $41,2^{\circ}$, inclinación pelviana $7,8^{\circ}$ y pendiente sacra $33,4^{\circ}$ (Figura 5 ). 


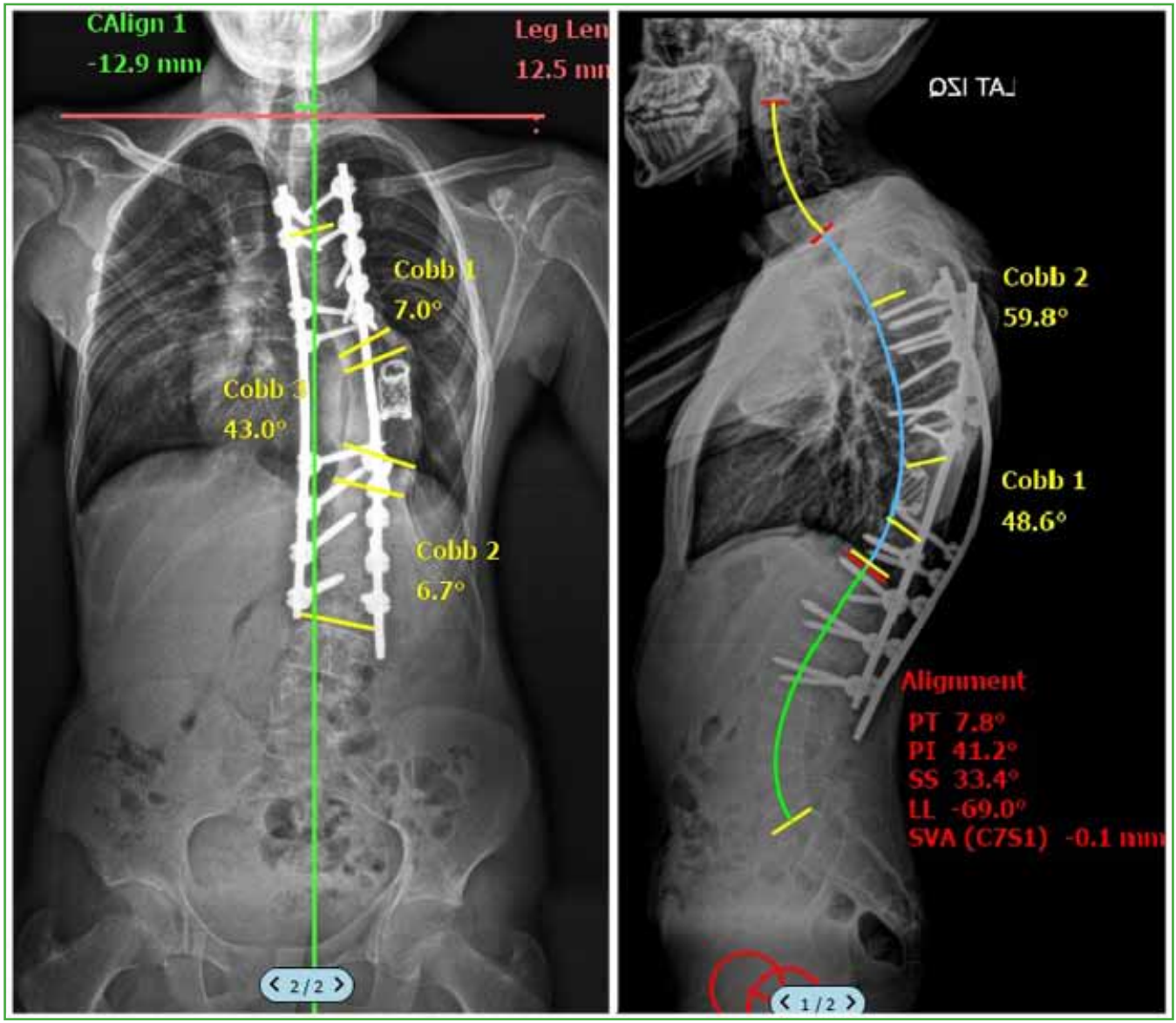

Figura 5. Espinograma posquirúrgico anteroposterior y lateral: ángulo de Cobb $43^{\circ}$, balance coronal $-12,9 \mathrm{~mm}$, cifosis segmentaria $48,6^{\circ}$, cifosis global $59,8^{\circ}$, balance sagital $-0,1 \mathrm{~mm}$.

El paciente no refiere dolor ni dificultad para deambular. La fuerza muscular es de $5 / 5$ en los miembros inferiores, la sensibilidad está conservada (Figura 6). Informa que comenzó la actividad física leve-moderada sin dificultad respiratoria. Al año, el puntaje SRS- 22 era de 4,5/5 (función 4,4/5, dolor 4,8/5; autoimagen 4,2/5; salud mental 4,4/5; satisfacción/insatisfacción con el manejo 5/5). Al segundo año de seguimiento, se incrementó la talla a 161 $\mathrm{cm}$ y el peso era de $48,9 \mathrm{~kg}$, con un índice de masa corporal de 18,8. Las medidas radiográficas a los dos años fueron: ángulo de Cobb $43,1^{\circ}$ de la curva torácica principal y $7^{\circ}$ de la curva torácica proximal, sin modificación respecto a la del año previo; balance coronal $+19,5 \mathrm{~mm}$ y hombros en balance. En el plano sagital, los hallazgos fueron: una cifosis segmentaria que se mantiene en $48,6^{\circ}$, como la cifosis T4-T12 en 59, $7^{\circ}$, balance sagital $0,1 \mathrm{~mm}$, lordosis lumbar $65,7^{\circ}$, incidencia pelviana $41,0^{\circ}$, inclinación pelviana $10,4^{\circ}$ y pendiente sacra $30,6^{\circ}$ (Figura 7 ). No se observa subsidencia de la malla de reemplazo corporal. El paciente practica actividad deportiva completa. El puntaje en el cuestionario SRS-22 fue de 4,7/5 (función 5/5; dolor 5/5; autoimagen 4,4/5; salud mental 4,2/5; satisfacción/insatisfacción con el manejo 5/5) a los dos años.

\section{DISCUSIÓN}

La escoliosis es una de las manifestaciones musculoesqueléticas más frecuentes en pacientes con neurofibromatosis, representa entre el $10 \%$ y el $64 \% .^{1,2,6,10,11} \mathrm{El}$ manejo de la deformidad no distrófica es similar al de una escoliosis idiopática. El desafío son las escoliosis distróficas, ya que, por sus características, tienden a ser de inicio temprano, con ángulos agudos que comprometen pocas vértebras, gran componente cifótico, añadido a las particularidades de dichas vértebras. ${ }^{2,12}$ Nuestro paciente cumple con los criterios de una escoliosis distrófica tal como se puede observar en la Figura 3. 


$$
\text { iii }
$$


En la bibliografía, se señala que este tipo de escoliosis, al ser muy agresiva, solo debe ser controlada si tiene $<20^{\circ}$, a diferencia de las escoliosis idiopáticas en las que se espera hasta los $40-50^{\circ}$. El manejo es quirúrgico luego de los $20^{\circ}$. Nuestro paciente tenía una cifoescoliosis severa de $107^{\circ}$ de Cobb coronal y $110,7^{\circ}$ de cifosis segmentaria que, en la actualidad, muy pocas veces se observan en la consulta, debido al manejo oportuno de estas deformidades, por eso la corrección se considera un verdadero reto no solo por el gesto quirúrgico, sino también por la alta tasa de complicaciones que conlleva este procedimiento. En las imágenes, se observa un gran desequilibrio en los planos coronal y sagital, con lo que el planteamiento debe ser la corrección de la deformidad y del balance en ambos planos.

La planificación quirúrgica incluyó una artrodesis vertebral posterior desde T4 hasta L3, con una corpectomía total posterior de T9 y reemplazo con malla de titanio, y osteotomías de Ponte en T7-T8 y T11-T12. El puntaje FOCOS $^{8}$ estratifica el riesgo quirúrgico sobre la base de los factores de riesgo y la planificación preoperatoria, el nivel de riesgo predice la aparición de complicaciones generales y neurológicas. Ciertos procedimientos, como las osteotomías y la resección de la columna vertebral, son factores predictivos independientes de complicaciones neurológicas posoperatorias. De acuerdo con este puntaje, que varía del 1 al 5 , a mayor puntaje, más alto es el riesgo de una complicación mayor, como la neurológica. En nuestro paciente, este puntaje fue de 5, con un $80 \%$ de riesgo de sufrir alguna complicación. Se optó por la cirugía en dos tiempos, ya que, según el estudio de dicho puntaje, el tiempo promedio quirúrgico con un puntaje 5 es de $367.2 \pm 169.1$ min y una pérdida sanguínea de 56,6 $\pm 24,2$ del volumen total; nuestro tiempo quirúrgico total fue de $1090 \mathrm{~min}$ (3 cirugías), con una pérdida sanguínea de $2900 \mathrm{~cm}^{3}$ que corresponde al 99\% del volumen total calculado. Si bien no hubo complicaciones mayores definitivas, sí se produjo una caída súbita de los potenciales motores y somatosensoriales durante la segunda intervención, lo que se clasificó como shock medular y se manejó según el protocolo NASCIS III; al despertar al paciente, la recuperación fue completa (ASIA E); esto concuerda con el riesgo de este puntaje, donde los puntajes 5 tienen hasta un $42,1 \%$ de cambios en el monitoreo neurofisiológico.

El manejo de las deformidades cifoescolióticas severas de la columna vertebral desde hace aproximadamente dos décadas se realiza solo por un abordaje posterior, conocido como resección vertebral de la columna, técnica popularizada por Lenke y cols., ${ }^{13}$ que tiene como objetivo la separación de la columna en dos extremos para luego unirlos y poder corregir la deformidad. La creación de este defecto segmentario genera tanta inestabilidad que exige una instrumentación provisional para evitar el daño medular. Es un procedimiento difícil y exigente que requiere de un profesional con amplia experiencia. La resección vertebral de la columna según la clasificación de $S_{c h w a b}{ }^{14}$ es el grado 5, que compromete la resección de una vértebra y sus discos subyacentes ${ }^{15}$ y requiere la colocación de un soporte anterior cuando el defecto es $>5 \mathrm{~mm}$. El tiempo promedio de esta cirugía es de 6.6-9 h, con una pérdida sanguínea estimada de $1600-2280 \mathrm{~cm}^{3} .^{13,16}$

Las complicaciones por este tipo de osteotomías varían del 58,5\% al 70,6\%. ${ }^{13,16}$ Auerbach y cols. ${ }^{17}$ identifican tres factores de riesgo para sufrir una complicación mayor: balance sagital $\geq 40 \mathrm{~mm}$, edad $\geq 60$ años y presencia de tres o más comorbilidades, también un tiempo quirúrgico $>8 \mathrm{~h}$ tuvo una asociación significativa. Durante la cirugía, las complicaciones más comunes son la pérdida de potenciales neurofisiológicos (27-29\%) y el sangrado $>2000 \mathrm{~cm}^{3}$. En el caso presentado, ocurrieron estas dos complicaciones, el sangrado global fue de $2900 \mathrm{~cm}^{3}(3$ intervenciones), pero el mayor sangrado por intervención fue de $1500 \mathrm{~cm}^{3}$. El grado de deformidad, la pérdida sanguínea superior a la estimada y la duración de la cirugía se asociaron con complicaciones intraoperatorias. ${ }^{13}$ El estudio de Lenke y cols. ${ }^{13}$ indica que no hay una gran diferencia entre las complicaciones perioperatorias y posoperatorias en cirugías de un solo tiempo versus las de dos o más tiempos, salvo por el tiempo quirúrgico más prolongado y el sangrado. Las complicaciones posoperatorias inmediatas más comunes son las respiratorias, como neumonías, derrame pleural, atelectasias. Las complicaciones mayores en este tipo de cirugías oscilan entre el 17\% y el 70,6\%, ${ }^{13,16,18-20}$ el 8-35\% son complicaciones neurológicas. Nuestro paciente no sufrió complicaciones mayores en el posoperatorio, ni secuelas neurológicas. La tasa de fracaso del material de osteosíntesis es del 14,3\% y, por lo general, ocurre dentro de los dos primeros años: ${ }^{21}$ los factores de riesgo descritos son un defecto anterior $>2 \mathrm{~cm}$, subsidencia de la malla de titanio $>5 \mathrm{~mm}$ e índice de masa corporal $>27$. Hasta el segundo año de seguimiento, el paciente no ha tenido dichas complicaciones, y se ha observado la integración de la artrodesis por tomografía.

Se recurre a la resección de la columna vertebral cuando la curva es rígida, con una flexibilidad $<35 \%,{ }^{1}$ o se busca una reducción de la curva $>40^{\circ}$, se logra una corrección de la escoliosis y la cifoescoliosis del 54-67,2\% y $60-90,9 \%$, respectivamente; ${ }^{20,22}$ en este caso, logramos una corrección de la cifosis segmentaria en $62,1^{\circ}(56,1 \%)$, de la escoliosis en $64^{\circ}(59,8 \%)$, y de la cifosis global en $70,5^{\circ}(54,1 \%)$, con una corrección de los balances coronal y sagital (mejoría 21,8 mm y 71,9 mm, respectivamente), sin mayores cambios en el segundo año de evolución. 
El cuestionario SRS-22 evalúa la satisfacción y el rendimiento del paciente antes de la cirugía y después de ella, ${ }^{7}$ e incluye cinco esferas clínicas: función, dolor, autoimagen, estado mental y satisfacción con el tratamiento. Permite tener una medida válida y fiable de la calidad de vida de los pacientes con escoliosis. ${ }^{23}$ También demuestra que la cirugía mejora el dolor, así como el resto de las esferas clínicas evaluadas hasta dos años luego de la cirugía. ${ }^{24}$ El puntaje global de nuestro paciente ha tenido una evolución favorable: 2,8 antes de la cirugía, 4,5 al año y 4,7 en el segundo año, con una mejoría importante de la autoimagen, la salud mental y la satisfacción con el tratamiento, esto indica el éxito clínico de acuerdo con la percepción del paciente.

\section{CONCLUSIONES}

La resección de la columna vertebral es una técnica quirúrgica potente y desafiante para el manejo de la deformidad cifoescoliótica compleja, necesaria para lograr el equilibrio espinal, pero no está exenta de complicaciones, sobre todo neurológicas y pulmonares, a veces, inevitables. La calidad de vida de este paciente ha mejorado de manera significativa.

Conflicto de intereses: Los autores no declaran conflictos de intereses.

ORCID de E. Morales Vásquez: https://orcid.org/0000-0002-0424-872X

ORCID de R. Macchiavello Falcon: https://orcid.org/0000-0002-3867-783X

\section{BIBLIOGRAFÍA}

1. Wang Z, Fu C, Leng J, Qu Z, Xu F, Liu Y. Treatment of dystrophic scoliosis in neurofibromatosis type 1 with onestage posterior pedicle screw technique. Spine J 2015;15(4):587.85. https://doi.org/10.1016/j.spinee.2014.10.014

2. Bersusky E. Deformidades vertebrales por neurofibromatosis. Rev Asoc Argent Ortop Traumatol 1999;64(4):263-9. https://www.aaot.org.ar/revista/1993_2002/1999/1999_4/640401.pdf

3. Coca Pérez A, Heredero JJ, Burgos J, Ferrero A, Aparicio-Meix JM. Neurofibromatosis tipo 1: escoliosis distrófica y tumor paravertebral. Rev Esp Pediatr 2004;60(3):243-5. https://pesquisa.bvsalud.org/portal/resource/pt/ibc-37743

4. Woodrow C, Clarke A, Amirfey R. Neurofibromatosis. Orthop Trauma 2015;29(3):206-10. https://doi.org/10.1016/j.mporth.2015.02.004

5. Patel N B, Stacy GS. Musculoskeletal manifestations of neurofibromatosis type 1. AJR Am J Roentgenol 2012;199(1):W99-106. https://doi.org/10.2214/AJR.11.7811

6. Crawford AH, Herrera-Soto J. Scoliosis associated with neurofibromatosis. Orthop Clin North Am 2007;38(4):55362. https://doi.org/10.1016/j.ocl.2007.03.008

7. Haher TR, Gorup JM, Shin TM, Homel P, Merola AA, Grogan DP, et al. Results of the Scoliosis Research Society instrument for evaluation of surgical outcome in adolescent idiopathic scoliosis. Spine 1999;24(14):1435-40. https://doi.org/10.1097/00007632-199907150-00008

8. Boachie-Adjei O, Yagi M, Sacramento-Dominguez C, Akoto H, Cunningham ME, Gupta M, et al. Surgical risk stratification based on preoperative risk factors in severe pediatric spinal deformity surgery. Spine Deform 2014;2(5):340-9. https://doi.org/10.1016/j.jspd.2014.05.004

9. Tejeda Barreras M. Esteroides en lesión medular postraumática aguda. Columna 2011;1(2):39-42. https://www.medigraphic.com/pdfs/columna/col-2011/col112d.pdf

10. Motono N, Kawaguchi M, Kawaharab N, Uramoto H. Case report of surgical treatment of scoliosis caused by neurofibroma located posterior mediastinum. Int J Surg Case Rep 2018;53:168-70. https://doi.org/10.1016/j.ijscr.2018.10.071

11. Tsirikos AI, Saifuddin A, Noordeen MH. Spinal deformity in neurofibromatosis type-1: diagnosis and treatment. Eur Spine J 2005;14(5):427-39. https://doi.org/10.1007/s00586-004-0829-7

12. Larson AN, Ledonio CGT, Brearley AM, Sucato DJ, Carreon LY, Crawford AH, et al. Predictive value and interrater reliability of radiographic factors in neurofibromatosis patients with dystrophic scoliosis. Spine Deform 2018;6(5):560-7. https://doi.org/10.1016/j.jspd.2018.02.011 
13. Lenke LG, Newton PO, Sucato DJ, Shufflebarger HL, Emans JB, Sponseller PD, et al. Complications after 147 consecutive vertebral column resections for severe pediatric spinal deformity. Spine (Phila Pa 1976) 2013;38(2):119-32. https://doi.org/10.1097/BRS.0b013e318269fab1

14. Schwab F, Blondel B, Chay E, Demakakos J, Lenke L, Tropiano P, et al. The comprehensive anatomical spinal osteotomy classification. Neurosurgery 2014;74(1):112-20. https://doi.org/10.1227/01.neu.0000462076.73701.09

15. Uribe JS, Schwab F, Mundis GM, Xu DS, Januszewski J, Kanter AS, et al. The comprehensive anatomical spinal osteotomy and anterior column realignment classification. J Neurosurg Spine 2018;29(5):565-75. https://doi.org/10.3171/2018.4.SPINE171206

16. Atici Y, Balioglu MB, Kargin D, Mert M, Albayrak A, Kaygusuz MA. Analysis of complications following posterior vertebral column resection for the treatment of severe angular kyphosis greater than $100^{\circ}$. Acta Orthop Traumatol Turc 2017;51(3):201-8. https://doi.org/10.1016/j.aott.2017.02.015

17. Auerbach JD, Lenke LG, Bridwell KH, Sehn JK, Milby AH, Bumpass D, et al. Major complications and comparison between 3-column osteotomy techniques in 105 consecutive spinal deformity procedures. Spine (Phila Pa1976) 2012;37(14):1198-210. https://doi.org/10.1097/BRS.0b013e31824fffde

18. Wang S, Aikenmu K, Zhang J, Qiu G, Guo J, Zhang Y, Weng, X. The aim of this retrospective study is to evaluate the efficacy and safety of posterior-only vertebral column resection (PVCR) for the treatment of angular and isolated congenital kyphosis. Eur Spine J 2017;26(7):1817-25. https://doi.org/10.1007/s00586-015-4344-9

19. Sacramento-Domínguez C, Yagi M, Ayamga J, Nemani VM, Akoto H, Mahmud R, et al. Apex of deformity for three column osteotomy. Does it matter in the occurrence of complications? FOCOS Spine Research Group. Spine $J$ 2015;15(11):2351-9. https://doi.org/10.1016/j.spinee.2015.07.010

20. Sheha ED, Kim HJ, Cunningham ME. Vertebral column resection for complex spinal deformity. Semin Spine Surg 2017;29(4):175-83. https://doi.org/10.1053/j.semss.2017.08.002

21. Wang H, Guo J, Wang S, Yang Y, Zhang Y, Qiu G, Zhang J. Instrumentation failure after posterior vertebral column resection in adult spinal deformity. Spine (Phila Pa 1976) 2017;42(7):471-8. https://doi.org/10.1097/BRS.0000000000001844

22. Sravisht Iyer, Venu M. Nemani, Han Jo Kim. A review of complications and outcomes following vertebral column resection in adults. Asian Spine J 2016;10(3):601-9. https://doi.org/10.4184/asj.2016.10.3.601

23. Climent JM, Bagó J, Rodríguez-Ruiz C, Sánchez-Raya J, Mulet S, Cholbi F. Nueva estrategia para mejorar la medida de la calidad de vida en la escoliosis idiopática: adición de la dimensión de deformidad percibida al cuestionario de la Scoliosis Research Society (SRS-22). Rehabilitación 2011;45(3):228-32. https://doi.org/10.1016/j.rh.2011.04.007

24. Merola AA, Haher TR, Brkaric M, Panagopoulos G, Mathur S, Kohani O, et al. A multicenter study of the outcomes of the surgical treatment of adolescent idiopathic scoliosis using the Scoliosis Research Society (SRS) outcome instrument. Spine (Phila Pa 1976) 2002;27(18):2046-51. https://doi.org/10.1097/00007632-200209150-00015 